\title{
Theorie und Praxis in der Entwicklungsforschung Einführung zum Themenheft
}

\section{Hermann Kreutzmann, Erlangen}

Die gesellschaftlichen und interdisziplinären Herausforderungen, die 1976 zur Gründung des Geographischen Arbeitskreises Entwicklungstheorien geführt haben, sind im wesentlichen gleich geblieben. Die Wahrnehmung einer Einkommenskluft zwischen armen und reichen Nationalstaaten und die Versuche, ihre Genese bzw. Verursachung zu erklären, boten den Anlass, nach dem Scheitern grobschlächtiger wachstumstheoretischer Erklärungsversuche neue Perspektiven zu erschließen und auf die Praxis anzuwenden. Der allgemeine Befund hat sich seither nicht signifikant zugunsten einer prinzipiellen Verlagerung verändert. Im Gegenteil:Die Entwicklungsschere (Abb.1) klafft weiterhin auseinander, die Einkommensunterschiede zwischen Arm und Reich könnten größer nicht sein und auch das Tempo der Auseinanderentwicklung nimmt stetig zu. Auch wenn die Perspektive auf Entwicklungsfragen sich in ihrem Schwerpunkt von einem eher auf den Nationalstaat als Bezugsgröße fixierten hin zu einem institutionen- und akteursbezogenen Multiebenen-Ansatz verschoben hat, so bleiben doch gewisse Grundfragen, ja die wichtigen und essentiellen, erhalten und harren weiterhin einer befriedigenden Beantwortung. Mancherorts scheinen sie in der diskursiven Vielfalt in Vergessenheit zu geraten bzw. im Meer divergierender Erklärungsansätze unterzugehen. Die Herausforderungen an die Praxis sind mit der Mannigfaltigkeit an Konzepten eng verflochten. Aus diesem Grunde hat Dieter SenghaAs, einer der einflussreichen Vordenker, Stichwortgeber und zudem Mitbegründer des Geographischen Arbeitskreises Entwicklungstheorien, vor einem «entwicklungstheoretischen Gedächtnisschwund» (SenghaAs 1996) gewarnt. Seine Mahnung erfolgte zu einem Zeitpunkt, als die Debatte über Abhängigkeitstheorien im Sande zu verlaufen schien und sich die Beispiele einer weltmarktintegrierten nachholenden Entwicklung eindrucksvoll durch die Prozesse in den sogenannten «Asiatischen Tigerstaaten» vermeintlich belegen liessen. Seinerzeit wurden daher Fragen zum «Ende der Dritten Welt» (Menzel 1992) und nach der Notwendigkeit von Entwicklungstheorie überhaupt erstmalig gestellt. Die Wahrnehmung differenzierter und unterschiedlicher Entwicklungspfade stellte tripolare und duale Konzepte monolithischen Blockdenkens in Frage. So diskutierte beispielsweise Deborah BräUtigam (1994) «What can Africa learn from Taiwan?» Diese und ähnliche globale Erklärungsversuche wurden im Hin- blick auf die Ungleichheiten von Partizipations- und Überlebenschancen kontrovers diskutiert (vgl. BERGER 1996; Воескн 1992; Elsenhans 1996; Hauck 1996; HeIn 1997, 1998; KöSsler \& Schiel 1996; Menzel 1995; Nuscheler 1997, 2000; Ortmanns 1996; PreSton 1996; Zapotoczky \& Gruber 1997). Dennoch gerieten die Grundfragen der theoretischen Debatte scheinbar ebenfalls aus dem Blickfeld. Dies zugunsten von weltmarktintegrierter Entwicklung, wie sie kurzfristig und allein von den «Tigerstaaten» vorgemacht zu sein schien, ferner von Theorien mittlerer Reichweite und Ansätzen, die stärker auf lokale und regionale Bedingungen abhoben. Letztere Entwicklungsfortschritte scheinen jenseits des Weltmarkts in Nischen eines adäquaten Umfelds und stärker in Individuen und Kleingruppen verankert zu sein. Globale Disparitätenentwicklung rückte in den Hintergrund, als die Auseinanderentwicklung innerhalb der «Dritten Welt» überkommene theoretische Erklärungsansätze zu diskreditieren schien. Das Unbehagen über Globaltheorien stimmte in den Missklang und die Frustration über die geringe bzw. verfehlte Wirkung von Entwicklungszusammenarbeit und die tatsächlich praktizierte Entwicklungspolitik ein. Das Nichtzustandekommen einer «Friedensdividende» aus den verfügbaren Mitteln nach Ende des Kalten Krieges zugunsten von Entwicklungsvorhaben nährte ebenso das Gefühl von Ohnmacht und Enttäuschung wie die Erkenntnis, dass die «weißen Flecken auf der Landkarte» (KAPLAN 1996; Menzel 2000) möglicherweise zunehmen.

Die Stagnation der Entwicklungsforschung schien ihren Lauf zu nehmen, als dankenswerterweise die konsequenten Sachwalter und unerschütterlichen Verfechter der entwicklungstheoretischen Debatte - die Redaktionsmitglieder der Zeitschrift «Peripherie» an frühere Bemühungen anknüpften (HEIN 1981), den Stand der Debatte in einem programmatischen Doppelheft unter dem Titel «Die Entwicklungstheorie ist tot - Es lebe die Theorie globaler Entwicklung!» (BRAND et al. 1997) beleuchteten und systematisch in einer Fachübersicht gliederten (Tab. 1). Hier wird eine mögliche Auffächerung der parallel laufenden Diskussionsstränge präsentiert, die sich mit Fragen der Entwicklung im engeren Sinne von Soziologie, Ökonomie und Geographie auseinandersetzen. Das Spektrum und die Spannweite wären durchaus noch erweiterbar, wie Reinhold Thiel (1999) in der Buchpublikation einer mehrjährigen in der Zeitschrift «Entwicklung und Zusammenarbeit» anregend geführten und von Franz Nuscheler (1998) immer 


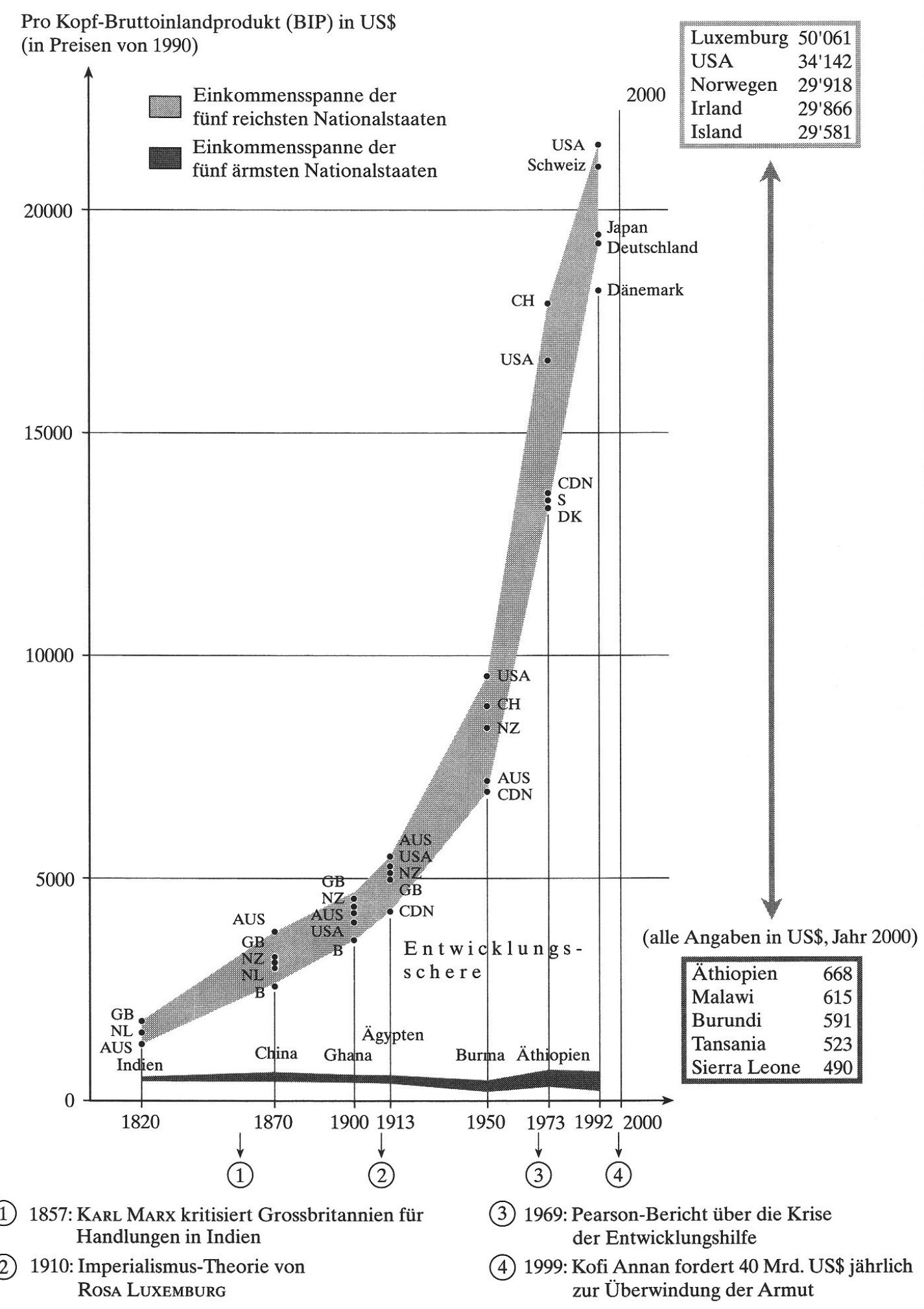

Abb. 1: Die Entwicklungsschere - Ausdruck einer wachsenden Kluft zwischen armen und reichen Nationalstaaten

The development gap - growing division between poor and rich nation-states

La fourchette du développement - Expression d'un clivage croissant entre Etats pauvres et Etats riches

Quelle: Kreutzmann (2002); United Nations Development Programme (UNDP, 2002) (veränd.) 
1. «Konventionelle» Entwicklungstheorie: von der Auseinandersetzung zwischen Modernisierungs- und Dependenztheorie zum «Ende der Grossen Theorie»

2. Theorien globaler Vergesellschaftung

3. Postindustrialismus, Postmoderne, end of history

- Postindustrielle Gesellschaften

- Postmoderne

- Finale Krise von Moderne und Modernisierungsprojekten

4. Dynamik und Transformation staatlicher Herrschaft

- Staatsanalyse, Nationalstaat

- Staat und Klassen

- Demokratisierung

5. Debatte um den Postfordimsus

- Fordismsus-These

- Konturen einer postfordistischen Formation

- Regulationstheoretische Ansätze

- Wirtschaftsgeographische und stadtsoziologische Ansätze

- Neo-Schumpeterianer

- Die These von der flexiblen Spezialisierung

- Die social structure of accumulation-Theorie

- Aspekte postfordistischer Regulation

- Die Veränderung von Staatlichkeit bzw. die Bedeutung der Globalisierung

- Die Internationalisierung des Kapitals, die Rolle der transnationalen Konzerne und internationaler Arbeitsteilung

- Die Verselbständigung des monetären Weltmarktes

6. Neue handels-, wachstums- und raumtheoretische Ansätze

- Handelstheoretische Ansätze

- Raumwirtschaftliche Ansätze

7. Nachholende Industrialisierung

8. Weltsystemtheorie

9. Internationale politische Ökonomie

10. Neo-Liberalismus

11. Umwelt und Entwicklung

12. Geschlecht und Entwicklung

- Übersichten über interkulturelle Frauenforschung

- Geschlecht, Arbeit, Ökonomie

- Frauen und Entwicklungspolitik

- Ethnologie und Geschlecht

- Frauenbewegungen und Entwicklung

- Feminismus und Ökologie

13. Bevölkerungsentwicklung und Entwicklung

- Die ökologische Argumentation

- Die ökonomische Theorie

- Die Theorie des demographischen Übergangs

- Familienplanung und Bevölkerungspolitik

Tab. 1: Von der Theorie der Unterentwicklung zur Theorie globaler Vergesellschaftung

From the theory of underdevelopment to the theory of global networks

De la théorie du sous-développement à celle de la sociétalisation globale

Quelle: zusammengestellt nach BRAND et al. (1997: 81-108) 
wieder stimulierten Erörterung des Themas gezeigt hat (Tab. 2a, 2b). Der Widerspruch zwischen nachholender und nachhaltiger Entwicklung, ja der Gegensatz von wachstumsorientierten bzw. allein darauf vertrauenden Strategien einerseits und die Begrenztheit von Ressourcen erkennenden und die Grenzen von Globalisierung aufzeigenden Entwicklungsbemühungen andererseits wird hier deutlich nachvollziehbar. Der Prozess und die Folgen der Globalisierung (Altvater \& Mahnkopf 2002; Beck 1998, 2002), Kontroversen über kulturalistisch begründete Modernisierung (vgl. Kreutzmann 2000) sowie die Hinwendung der sozialwissenschaftlichen Debatte zu verstärkt handlungstheoretisch begründeten Ansätzen bildeten die Zäsur und gleichzeitig den Versuch, Parallelentwicklungen konstruktiv zu erfassen und nicht das Eine durch das Andere zu verdrängen. Denn früher gestellte Grundfragen, wie die nach der Entwicklungsschere bzw. nach Abhängigkeitskonstellationen und Chancen autozentrierter Entwicklung, waren ja keinesfalls hinreichend und abschließend beantwortet bzw. ad acta gelegt worden. Im Gegenteil, die Fragen nach begrifflicher Ausfüllung und nach den analytisch-prozessualen und strategischen Grundbedingungen von Entwicklung und Unterentwicklung sind virulent wie eh und je. Die Austauschbeziehungen unter Globalisierungsbedingungen haben im Zuge von Deregulierung, Entstaatlichung, Machtzuwachs transnationaler Konzerne trotz Verlautbarungen des Gegenteils eher zu einer Verschärfung des «Entwicklungsklimas» und zu einem weiteren Auseinanderklaffen der Entwicklungsschere geführt. An dieser Stelle sind die bemerkenswerten Einschätzungen des Nationalökonomen JosEPH STIGLITZ (2002) zu erwähnen, der, basierend auf eigenen Erfahrungen als handelnder Akteur in der US-amerikanischen Regierung und in der WeLtBank, eine vehemente Kritik am INTERNATIONALEN WÄHRUNGS-FONDS (IWF) vorgetragen hat und zu einer Reorientierung der Entwicklungsziele der WeLtbank aufruft. In einer längst in Vergessenheit geratenen Deutlichkeit sind Brüche und Widersprüche gesellschaftlich wahrnehmbar geworden, wie es die leidenschaftlich und mittlerweile auch medienwirksam geführten Auseinandersetzungen zwischen Globalisierungsbefürwortern und -gegnern belegen (vgl. auch GREFE, GREFFRATH \& Schumann 2002). Letztere fordern im Sinne von PIERRE Bourdieu (2001) eine neue «europäische soziale Bewegung», die damit dem Thema menschengerechter Entwicklungschancen und Partizipation (HELLER 2002; RAUCH 2002) wieder einen höheren gesellschaftlichen Stellenwert einräumen könnte.

Die Schlussfolgerung, die sich aus diesen sehr verkürzt präsentierten Überlegungen ableiten lässt, ist, dass nach den frühen Moden der angeblich alles und manchmal doch zu wenig erklärenden Globaltheorien gegenwärtig die Erkenntnisse in der «mittleren Reich- weite», wenn nicht allein im individuellen, alltagsweltlichen Handlungsraum, der multidimensional verflochten ist, gesucht werden. In der Entwicklungspraxis dominieren eher pragmatische Ansätze, die zu einem grossen Teil im entpolitisierten Rahmen zu agieren vorgeben bzw. einer zweifelhaften politischen Konditionalität folgen. Gleichzeitig ist in jüngster Zeit das öffentliche und akademische Interesse an Entwicklungsfragen wieder gestiegen. Aufgaben für die Gegenwart und Zukunft stellen dar:

- gesellschaftliche Ungleichheit und die Belastung friedvoller Austauschbeziehungen durch armutsbetonte Konfrontation,

- internationale Mobilität in einer sich zumindest für Teilgruppen partiell entgrenzenden Welt,

- Aufbau neuer Barrieren, die Schaffung von gated communities, von Wohlstands- und Armuts-Ghettos, - Exklusion breiter Bevölkerungsschichten von der Teilhabe an sozialer Infrastruktur und Wohlfahrt.

Diese Herausforderungen sind Teile des Diskurses, u.a. im Feuilleton und Wirtschaftsteil überregionaler Zeitungen. An dieser Stelle sei nur auf die allmonatliche Berichterstattung in der Le Monde Diplomatique verwiesen, die eine Diskussionsplattform für eine sozialwissenschaftlich geprägte, kritische Wahrnehmung globaler Konfliktfelder bietet.

\section{Geographische Entwicklungsforschung im Zeichen der Globalisierung}

Welche Konsequenzen erwachsen aus der Ausdifferenzierung der entwicklungstheoretischen Debatte für die geographische Entwicklungsforschung? Verschiedene Standortbestimmungen wurden unternommen (vgl. Coy 2000). Der Gründer des Geographischen Arbeitskreises Entwicklungstheorien, FRed ScHOLZ, hat in den letzten Jahren vermehrt die Herausforderungen der Globalisierung und einer veränderten sozialwissenschaftlichen Paradigmenbildung aufgegriffen und in mehreren Beiträgen breitenwirksam präsentiert (Scholz 2000a, 2000b, 2001, 2002). In seiner «Theorie der fragmentierenden Entwicklung» versucht er nachzuweisen, dass das Modell der nachholenden Entwicklung unter bestehenden globalen Grenzbedingungen nicht umsetzbar sei, sich ein Gegensatz der Lebensverhältnisse im globalen Maßstab verfestige und gleichfalls auf kleinräumiger Ebene in unmittelbarer Nachbarschaft wiederfinden ließe. Eine solche Erkenntnis relativiert die «Perspektiven des Südens» grundlegend und stimmt ein in den Kanon derer, die die «Ideologie der Entwicklung» (Latouche 2001; MenZeL 2000) an den Pranger stellen. Was bleibt zu tun?

Eine Reflexion über Bedeutungsinhalt und normative Setzungen, die mit Entwicklung in Theorie und Praxis verknüpft werden, ist nötig und in den letzten Jahren 


\begin{tabular}{|c|c|c|}
\hline Theorie & Inhalt & Anmerkungen \\
\hline $\begin{array}{l}\text { Wachstumstheorie } \\
\text { (Dualismustheorie, } \\
\text { Modernisierungstheorie) }\end{array}$ & $\begin{array}{l}\text { Gleichsetzung von Wachstum } \\
\text { und Entwicklung } \\
\text { Zufuhr von Kapital wird in } \\
\text { unterentwickelten Ländern } \\
\text { Wachstum induzieren } \\
\text { nachholende Entwicklung ist } \\
\text { so möglich }\end{array}$ & $\begin{array}{l}\text { Scheitern wurde in den } \\
\text { 1960er Jahren erkannt } \\
\text { (Entwicklungsschere) } \\
\text { vorherrschende } \\
\text { Entwicklungsstrategie } \\
\text { Ausnahme: ostasiatische } \\
\text { Tigerstaaten }\end{array}$ \\
\hline Dependenztheorie & $\begin{array}{l}\text { strukturelle Ungleichheit } \\
\text { charakterisiert das Verhältnis } \\
\text { zwischen Entwicklungs- } \\
\text { (Peripherie) und } \\
\text { Industrieländern } \\
\text { (Metropolen) } \\
\text { Zentrum und Peripherie } \\
\text { stehen in einem } \\
\text { asymmetrischen } \\
\text { Abhängigkeitsverhältnis } \\
\text { Abkopplung erlaubt } \\
\text { eigenständige } \\
\text { (autozentrierte) Entwicklung }\end{array}$ & $\begin{array}{l}\text { durchaus empirisch belegbar, } \\
\text { vor allem im historischen } \\
\text { Kontext auf der Ebene von } \\
\text { Nationalstaaten } \\
\text { (Entwicklungsschere) } \\
\text { Weltwirtschaftsbeziehungen } \\
\text { haben starke und schwache } \\
\text { Akteure, die nicht zwingend } \\
\text { Nationalstaaten zu sein haben } \\
\text { Scheitern wurde nicht zuletzt } \\
\text { durch fehlende Vorbilder/ } \\
\text { Beispiele konstatiert }\end{array}$ \\
\hline Zentralplanungsmodell & $\begin{array}{l}\text { Modell des «real } \\
\text { existierenden Sozialismus» } \\
\text { (zweite Welt) } \\
\text { Verstaatlichung der gesamten } \\
\text { Wirtschaft: Entwicklung über } \\
\text { Schwerindustrialisierung } \\
\text { zentralstaatlich gelenkte } \\
\text { Planwirtschaft }\end{array}$ & $\begin{array}{l}\text { endet mit dem Ende der } \\
\text { «zweiten Welt» } \\
\text { (Entwicklungsschere) } \\
\text { Modell scheitert mit der } \\
\text { Postindustrialisierung } \\
\text { wird zum Milleniumswechsel } \\
\text { in fast allen Staaten } \\
\text { aufgegeben } \\
\text { (Transformationsstaaten) }\end{array}$ \\
\hline Neoliberales Modell & $\begin{array}{l}\text { Entfesselung der Märkte } \\
\text { Einschränken der } \\
\text { Staatsfunktion } \\
\text { Herstellung günstiger } \\
\text { Marktbedingungen }\end{array}$ & $\begin{array}{l}\text { Ostasien- und Lateinamerika- } \\
\text { Krise überzeugten viele vom } \\
\text { Scheitern } \\
\text { Spielart der Renaissance der } \\
\text { Wachstumstheorien } \\
\text { mainstream-Ökonomen sehen } \\
\text { es als alternativlos an }\end{array}$ \\
\hline $\begin{array}{l}\text { Modell staatlich } \\
\text { gelenkter } \\
\text { Privatwirtschaft }\end{array}$ & $\begin{array}{l}\text { «Gänseflug-Modell» } \\
\text { (Kaname in den 1930er } \\
\text { Jahren) der ostasiatischen } \\
\text { Staaten } \\
\text { kaskadenartige Entwicklung } \\
\text { basierend auf hoher interner } \\
\text { Sparquote }\end{array}$ & $\begin{array}{l}\text { war erfolgreich, solange } \\
\text { unter nationalstaatlicher } \\
\text { Protektion } \\
\text { Scheitern mit Öffnung für das } \\
\text { Modell des «Washington } \\
\text { Consensus» }\end{array}$ \\
\hline
\end{tabular}

Tab. 2a: Ökonomische Deutungsmodelle, die wirtschaftliches Wachstum analysieren und nachholende Entwicklung prognostizieren.

Economic evaluation models that analyse economic growth and forecast catch-up development. Modèles économiques qui analysent la croissance économique et qui pronostiquent le rattrapage en matière de développement.

Quelle: THIEL (1999) (veränd., ergänzt) 


\begin{tabular}{|c|c|c|}
\hline Theorie & Inhalt & Anmerkungen \\
\hline $\begin{array}{l}\text { Konzept der kulturellen } \\
\text { Bedingtheit von } \\
\text { Entwicklung }\end{array}$ & $\begin{array}{l}\text { Entwicklung basiert auf } \\
\text { religiös fundierter } \\
\text { Wirtschaftsethik } \\
\text { Zusammenhang zwischen } \\
\text { wirtschaftlicher } \\
\text { Entwicklung und religiös- } \\
\text { kulturellem Wertesystem } \\
\text { Untersuchung } \\
\text { außerwirtschaftlicher } \\
\text { Zusammenhänge liefert } \\
\text { Aufklärung über Wirkung } \\
\text { wirtschaftlicher Faktoren } \\
\text { an definiertem Ort und zu } \\
\text { bestimmter Zeit } \\
\text { Wirtschaftsethnologie }\end{array}$ & $\begin{array}{l}\text { protestantische Ethik } \\
\text { Konfuzianismus-Debatte, } \\
\text { asiatische Werte, bislang } \\
\text { fehlende Verknüpfung von } \\
\text { Makroökonomie und } \\
\text { Wirtschaftsethnologie } \\
\text { Kultur-Debatte, Einbettung } \\
\text { von Wirtschaft in Kultur } \\
\text { und Gesellschaft. } \\
\text { Kritik: überkomplexer } \\
\text { Ansatz, menschliches } \\
\text { Handeln findet nicht } \\
\text { gebührende } \\
\text { Berücksichtigung, } \\
\text { Erklärungspotential ist } \\
\text { bislang nicht ausgeschöpft } \\
\text { Kolonialismus-Schock der } \\
\text { Ethnologie wurde bislang } \\
\text { nicht überwunden }\end{array}$ \\
\hline $\begin{array}{l}\text { Konzept der } \\
\text { nachhaltigen } \\
\text { Entwicklung }\end{array}$ & $\begin{array}{l}\text { Neudefinition von } \\
\text { Entwicklungszielen für die } \\
\text { Zukunft } \\
\text { Aufzeigen eines } \\
\text { alternativen } \\
\text { Entwicklungspfades } \\
\text { Kritik an } \\
\text { Wachstumstheorien } \\
\text { Einschränkung statt } \\
\text { Expansion: Vermeidung, } \\
\text { um die Entwicklungs- } \\
\text { fähigkeit für die Zukunft } \\
\text { zu erhalten }\end{array}$ & $\begin{array}{l}\text { vornehmlich ein } \\
\text { strategisches Konzept } \\
\text { basierend auf } \\
\text { Ressourcenausstattung } \\
\text { normativer Ansatz aus } \\
\text { ökologischen Überlegungen } \\
\text { entstanden, der bislang nicht } \\
\text { erklären kann, wie ein } \\
\text { Wirtschaftssystem } \\
\text { funktionieren könnte, das } \\
\text { auf Ressourcenschonung } \\
\text { basiert } \\
\text { in jüngster Zeit erweitert } \\
\text { um soziale Gerechtigkeit }\end{array}$ \\
\hline
\end{tabular}

Tab. 2b: Über ökonomische Ansätze hinausgreifende Theorien

Theories that extend beyond economic aspects.

Théories dépassant des approches économiques

Quelle: THIEL (1999) (veränd., ergänzt)

vermehrt Schwerpunkt der Diskussion im Geographischen Arbeitskreis Entwicklungstheorien. Im Jahre 2000 standen in Zürich Themen zu «institutionellen Regelungen im Entwicklungsprozess» im Zentrum der Diskussion, die nicht ohne weiteres eindeutig einer Facette der Übersicht (Tab. 1) zuzuordnen wäre (vgl. Geographica Helvetica 2001, 56, 1 - Themenheft «Institutionelle Regelungen im Entwicklungsprozess»). Konfliktträchtige Auseinandersetzungen um die Teilhabe an Gemeinschaftseigentum und um Rechtstitel an Ressourcen sind einerseits damit gemeint, andererseits auch das, was die WeLtbank (2002) in ihrem jüngsten Weltentwicklungsbericht unter dem Titel «Institutionen für Märkte schaffen» als die treibenden Kräfte für Fortschritt ansieht.

Für den im April 2002 in Erlangen durchgeführten Workshop wurde das Motto «Theoriediskurse versus lokale Anwendung von Konzepten? Relevanz und Defizite von Ansätzen zur Überlebenssicherung» gewählt. Dem Wunsch zahlreicher Arbeitskreismitglieder entsprechend war eine stärkere Verknüpfung von Theorie und Praxis gefordert bzw. eine Auslotung des theoretischen Gehalts von in der Praxis zur Anwen- 
dung kommenden entwicklungsstrategischen Konzepten von Nöten. Aus den Diskussionszusammenhängen der auf diese Bipolaritäten eingestimmten Arbeitsgruppen stammen die im folgenden abgedruckten Beiträge. Ihre breite thematische Streuung liegt an der Tatsache, dass in den Arbeitsgruppendiskussionen eine weitere Ausdifferenzierung des Themas vorgenommen wurde. Unterschiedliche Positionen zum livelihoodAnsatz und vor allem seines theoretischen und operationalisierbaren Gehalts erfuhren frühzeitig Akzentuierungen und eröffneten eine breit angelegte und grundsätzlichere Diskussion. Folglich sind die Blickrichtungen der hier präsentierten Beiträge recht unterschiedlich, decken damit aber auch die Vielfalt der vorgenommenen Positionierungen ab.

Im einleitenden Beitrag werden von THOMAs DöRfLer, Olivier Grefe und Detlef Müller-Mahn prinzipielle und kritische Fragen an die Entwicklungstheorie und ihr Betätigungsfeld überhaupt gerichtet. Die Autoren loten die Anwendbarkeit des von Pierre Bourdieu entwikkelten Habitus-Konzeptes aus und skizzieren mögliche Felder einer entwicklungstheoretischen Betrachtung, die über den individuumszentrierten Handlungsspielraum GidDENsscher Prägung hinaus den gesellschaftlichen Referenzrahmen stärker einbezieht.

Gesellschaftlicher Transformation unter den Bedingungen der sich verschärfenden Globalisierung widmet sich SABINE TRÖGER. Sie lotet anhand der These über die «Dualität der Struktur» die Spielräume für eine Modifikation und auch Umkehrung von vorgefundenen Strukturen durch die Handlungsträger aus. Exemplifiziert werden ihre Überlegungen anhand des «dualen Landrechts» in Tansania. Die selektiven Nutzer hinzugewonnener Spielräume verkörpern die Gewinner der Globalisierung, während die machtlosen Armen bei schrumpfenden Freiheitsgraden verwundbarer geworden sind und zu ihren Verlierern zählen.

Auf der Basis empirischer Praxiserfahrung, die sich $\mathrm{zu}$ theoretischen Überlegungen in Beziehung setzen lässt, wird das Spektrum aktueller Debatten ausgeleuchtet. Theo RAuch lässt die Diskussionen über das Pro und Kontra entwicklungspolitischen Engagements und -praktischer Interventionen Revue passieren, um aus dem wahrgenommenen Dilemma einer angebotsorientierten Entwicklungsofferte zu einem Analyserahmen zu gelangen, der hier als eine Vier Ebenen-Interventionsstrategie vorgestellt wird. Es geht um eine angemessene Intervention in klar definierten Feldern, die in einer Matrix dargestellt wird.

Unter dem Stichwort «Risiko» und seiner Beziehung zur Existenzgefährdung bzw. -absicherung beziehen sich Fred Krüger und Elísio Macamo auf das Analyseschema des livelihood-Ansatzes und erweitern ihn um soziologische Kategorien, die sich u.a. an ULRICH BECKs Überlegungen zur Risikogesellschaft anlehnen. Der gesellschaftliche Umgang mit Unsicherheit und die Einschätzung von Gefahren werden aus der strukturellen Tatsachenverhaftung in einen probabilistischen Kontext personalisierter Beurteilungen gehoben, der den Rahmen für menschliches Handeln abgibt. Die Autoren sehen hier Ansatzpunkte für die Übertragung in die Praxis.

«Können Öko-Siegel einen neuen Handlungsspielraum für kleinbäuerliche Kaffeeproduzenten schaffen?» fragt Claudia MaYer und stellt die lokalen und produktionsbedingten Begrenzungen einer Warenbeziehung heraus, die mit hoher Akzeptanz bei darauf eingestimmten Käuferschichten des Nordens besetzt ist. Die Einbettung der lokalen Akteure nicht nur in Weltmarktbeziehungen, sondern vor allem auch die lokale und regionale Ausfüllung des durch die Einführung von Öko-Siegeln entstandenen Handlungsspielraums werden hier thematisiert unter Beachtung vielfältiger Rahmenbedingungen.

Die Bandbreite der Beiträge spiegelt segmentär die Spannweite der aktuellen Diskussion und Varianz thematischer Foci innerhalb der geographischen Entwicklungsforschung wider. Sowohl für die entwicklungstheoretische Debatte als auch im Hinblick auf empirische Untersuchungen und praktische Interventionen sind weitere Anstöße und Kontroversen nötig. Die hier zusammengestellten Beiträge möchten für die Diskussion darüber Impulse geben.

\section{Literatur}

Altvater, E. \& B. Mahnkopf (2002): Globalisierung der Unsicherheit. Arbeit im Schatten, schmutziges Geld und informelle Politik. - Münster: Westfälisches Dampfboot.

BECK, U. (Hrsg.) (1998): Perspektiven der Weltgesellschaft. - Frankfurt am Main: Suhrkamp.

Beck, U. (Hrsg.) (2002): Macht und Gegenmacht im globalen Zeitalter. Neue weltpolitische Ökonomie. Frankfurt am Main: Suhrkamp.

BERgER, J. (1996): Was behauptet die Modernisierungstheorie wirklich - und was wird ihr bloß unterstellt? In: Leviathan. Zeitschrift für Sozialwissenschaft 24, 1: 45-62.

Bоескн, A. (1992): Entwicklungstheorie: eine Rückschau. - In: Nohlen, D. \& F. Nuscheler (Hrsg.): Handbuch der Dritten Welt 1, Bonn: 110-130.

Bourdieu,P.(2001): Gegenfeuer 2: Für eine europäische soziale Bewegung. - Konstanz: UVK-Verlagsgesellschaft.

BräUtigam, D. (1994): What can Africa learn from Taiwan? - In: The Journal of Modern African Studies 32: 111-138. 
BRAND, R. et al. (1997): Von der Theorie der Unterentwicklung zur Theorie globaler Vergesellschaftung (Fachübersicht). - In: Peripherie 17, 65-66: 81-108.

Coy, M. (2000): Aufgaben der Geographie für Entwicklungsländerforschung und Entwicklungspolitik. In: Blotevogel, H.H., OssenbrügGe, J. \& G. Wood (Hrsg.): Lokal verankert - weltweit vernetzt. - Stuttgart: Franz Steiner Verlag: 46-58.

Elsenhans, H. (1996): Kein Ende der großen Theorie. - In: Asien - Afrika - Lateinamerika 24, 2: 111-146.

Geographica Helvetica (2001): Themenheft «Institutionelle Regelungen im Entwicklungsprozess». - In: Geographica Helvetica 56, 1.

Grefe, C., Greffrath, M. \& H. Schumann (2002): Attac. Was wollen die Globalisierungsgegner?-Berlin: Rowolth.

HAUCK, G. (1996): Evolution, Entwicklung, Unterentwicklung. - Frankfurt am Main: Iko-Verlag.

HeIN, W. (1981): Fachübersicht:Zur Theorie der Unterentwicklung und ihrer Überwindung. - In: Peripherie 5/6: 64-91.

HeIN, W. (1997): Das «Scheitern der Großen Theorie», der Globalisierungsprozess und die Krise nationaler Gesellschaften. - In: Peripherie 17, 65-66: 109-132.

Hein, W. (1998): Unterentwicklung - Krise der Peripherie. Phänomene, Theorien, Strategien. - Opladen: Leske \& Budrich.

Heller, P. (2002) Den Staat in Bewegung bringen. Die Politik der demokratischen Dezentralisierung in Kerala, Südafrika und Porto Alegre. - In: Peripherie 87: 337-377.

KaPLAN, R. (1996): The ends of the earth. From Togo to Turkmenistan, from Iran to Cambodia. A journey to the frontiers of the anarchy. - New York: Vintage.

KöSSLER, R. \& T. Schiel (1996): Auf dem Weg zu einer kritischen Theorie der Modernisierung. - Frankfurt am Main: Iko-Verlag.

Kreutzmann, H. (2000): Von der Modernisierungstheorie zum clash of civilizations: Gemeinsamkeiten und Widersprüche strategischer Entwicklungsvorstellungen. - In: Diekmann, I., KrÜGer, P. \& J.H. Schoeps (Hrsg.): Geopolitik. Grenzgänge im Zeitgeist. Band 1.11890 bis 1945, Band 1.21945 bis zur Gegenwart. - Potsdam: Verlag für Berlin-Brandenburg: 453-477.

Kreutzmann, H. (2002): Zehn Jahre nach Rio - (Wieder-)Entdeckung der Armut oder Entwicklungsfortschritte im Zeichen der Globalisierung? - In: Geographische Rundschau 54, 10: 58-63.

LATOUCHE, S. (2001): Die Ideologie der Entwicklung. Abrechnung mit einem hoffnungslosen Begriff. - In: Le Monde Diplomatique. Beilage der Tageszeitung vom 15. Juni 2001: 16.

Le Monde Diplomatique. - Monatlich erscheinend, Paris (deutsche Ausgabe als Beilage der «Tageszeitung» und als Einzelausgabe, Berlin).

Menzel, U. (1992): Das Ende der Dritten Welt und das
Scheitern der großen Theorie. - Frankfurt am Main: Suhrkamp.

Menzel, U. (1995): Geschichte der Entwicklungstheorie. Einführung und systematische Bibliographie. - = Schriften des Deutschen Übersee-Instituts Hamburg 31, Hamburg: Selbstverlag.

Menzel, U. (2000): Das Ende der einen Welt oder Die weißen Flecken auf der Landkarte nehmen wieder zu. - In: BETZ, J. \& S. BRÜNE (Hrsg.): Jahrbuch Dritte Welt. - München: Beck: 19-31.

NusCHELER, F. (1997): Entwicklungstheorien im Widerspruch. - In: Zapotoczky, K. \& P. C. Gruber (Hrsg.): Entwicklungstheorien im Widerspruch. Plädoyer für eine Streitkultur in der Entwicklungspolitik. - Frankfurt am Main, Wien: Brandes \& Apsel/Südwind: 20-34. NusCHeler, F. (1998): Warum brauchen wir Entwicklungstheorien? - In: Entwicklung und Zusammenarbeit 39, 11:284-287.

NusCheleR, F. (2000): Vom (großen) Nutzen und (kleinen) Elend der Komparatistik in der Entwicklungstheorie. - In: Menzel, U. (Hrsg.): Vom ewigen Frieden und vom Wohlstand der Nationen. - Frankfurt am Main: Suhrkamp: 467-492.

Ortmanns, B. (1996): Ist die Dritte Welt wirklich am Ende? Zur Kritik von Entwicklungstheorien. - In: Aus Politik und Zeitgeschichte 12 v. 15.3.1996: 27-36.

Peripherie (1997): Themenheft «Die Entwicklungstheorie ist tot - Es lebe die Theorie globaler Entwicklung». - In: Peripherie 17, 65-66.

Preston, P.W. (1996): Development Theory. An introduction. - Oxford: Blackwell Publishers.

RAUCH, T. (2002): Partizipation in der Entwicklungszusammenarbeit auf dem Weg von der Spielwiese hin zum demokratischen Recht für alle. - In: Peripherie 22, 88: 496-522.

Scholz, F. (2000a): Perspektiven des «Südens» im Zeitalter der Globalisierung. - In: Geographische Zeitschrift 88, 1: 1-20.

Scholz, F. (2000b): Globalisierung versus Fragmentierung. Eine regionalwissenschaftliche Herausforderung? - In: Nord-Süd aktuell 14, 2: 255-271.

Scholz, F. (2001): Nachholende Entwicklung: Kein Entwicklungsparadigma mehr im Zeitalter der Globalisierung. - In: epd-Entwicklungspolitik 11/12: 35-37.

Scholz, F. (2002): Globalisierung und Entwicklung. Die Theorie der «fragmentierten Entwicklung». - In: Geographische Rundschau 54, 10: 6-11.

SENGHAAS, D. (1996): Wider den entwicklungstheoretischen Gedächtnisschwund. Die Auswege aus der Armut sind bekannt. - In: Entwicklung und Zusammenarbeit 37, 9:236-237.

Stiglitz, J. (2002): Die Schatten der Globalisierung. Berlin: Siedler Verlag.

ThIEL, R.E. (Hrsg.) (1999): Neue Ansätze zur Entwicklungstheorie. - Bonn: Selbstverlag der Deutschen Stiftung für internationale Entwicklung (DSE).

United Nations Development Programme (UNDP) 
(2002): Bericht über die menschliche Entwicklung 2002. Stärkung der Demokratie in einer fragmentierten Welt. - Bonn: UNO-Verlag.

WeltBank (2002): Weltentwicklungsbericht 2002. Institutionen für Märkte schaffen. - Bonn: UNO-Verlag.

Zapotoczky, K. \& P.C. Gruber (Hrsg.) (1997): Entwicklungstheorien im Widerspruch. Plädoyer für eine Streitkultur in der Entwicklungspolitik. - Frankfurt am Main, Wien: Brandes \& Apsel/Südwind.

\section{Zusammenfassung: Theorie und Praxis in der Ent- wicklungsforschung - Einfïhrung zum Themenheft}

Unzufriedenheit mit dem Erklärungsanspruch konventioneller globaler Entwicklungstheorien hat bei manchen Protagonisten der Disziplin zu einer Redundanzhaltung geführt, während andere Fachvertreter eine größere Notwendigkeit denn je zu vermeinen spüren. Seit einigen Jahren wird die Debatte einerseits systematischer und andererseits facettenreicher geführt. Der Beitrag zeigt einige Entwicklungsstränge auf und betont die Persistenz wichtiger Grundfragen der Entwicklungsforschung am Beispiel der sich öffnenden Entwicklungsschere. Sie symbolisiert globale Fehlentwicklungen und stellt zugleich eine bleibende Herausforderung an Forschung, Politik und Entwicklungspraxis dar.

\section{Summary: Theoretical and Practical Approaches in Development Research - Introduction to the special issue}

Growing discontent with the explanatory assertions of conventional global development theories led some protagonists of the field to complain of gross redundancy while other representatives of our discipline felt there was a greater demand for theory than ever before. In recent years the debate has become more systematic while the spectrum of conceptual approaches has grown. The article describes several recent developments, highlighting the persistance of important basic questions in research development like the widening gap between poor and rich countries. The symbolism of the wealth gap is taken to underline the continued challenge faced by academic research, political action and implementing agencies.
Résumé: Théorie pratique dans la recherche sur le développement - Introduction au cahier thématique Le mécontentement, qui s'est manifesté à l'occasion des approches faites par les théories conventionnelles relatives au développement global, a conduit maint protagoniste de la discipline à un comportement redondant, pendant que d'autres spécialistes ont ressenti plus que jamais la nécessité de faire progresser la réflexion théorique. Depuis quelques années, le débat est mené à la fois de manière plus systématique et plus variée. La présente contribution montre quelques axes de développement et met l'accent sur la persistance de questions fondamentales dans la recherche sur le développement, à l'exemple de l'élargissement de la fourchette du développement. Celle-ci symbolise des développements globaux marqués et continue à interroger la recherche, la politique et la pratique du développement.

\section{Didaktische Hinweise}

- Hat die Globalisierung zur Öffnung der weltweiten Entwicklungsschere beigetragen?

- Welche Herausforderungen stellen sich auf dem Feld der Entwicklung in Gegenwart und Zukunft?

- Bieten Entwicklungstheorien Erklärungen und Antworten auf die wachsende Kluft zwischen armen und reichen Nationalstaaten?

- Welches Spektrum decken aktuelle Forschungsansätze in der Entwicklungsforschung ab?

Prof. Dr. Hermann Kreutzmann, Institut für Geographie der Universität Erlangen, Kochstrasse 4/4, D-91054 Erlangen.

e-mail: hkreutzm@geographie.uni-erlangen.de

Manuskripteingang/received/manuscrit entré le 29.1.2003 